\title{
Lock-in Thermography for analyzing solar cells and failure analysis in other electronic components
}

\author{
by O. Breitenstein* and S. Sturm**
}

\author{
* Max Planck Institute of Microstructure Physics, Weinberg 2, D-06120 Halle, Germany, breiten@mpi-halle.mpg.de \\ **InfraTec GmbH, Gostritzer Str. 61-63, D-01217 Dresden, Germany, s.sturm@infratec.de
}

\begin{abstract}
Lock-in thermography (LIT) is a dynamic variant of infrared thermography, where local heat sources are periodically pulsed and amplitude and phase images of the surface temperature modulation are obtained. If used in electronic device testing, this method enables the localization of very weak local heat sources below the surface. This contribution reviews the basics and application of LIT for local efficiency analysis of solar cells and for failure analysis in other electronic components like bare and encapsulated integrated circuits. In both application fields LIT has established as a reliable and easy-to-use standard method for failure analysis.
\end{abstract}

\section{Introduction}

Lock-in thermography (abbreviated LIT or LT), which is the phase-locked detection of local periodic surface temperature modulations in a device, was proposed in 1988 for investigating micro-cracks in an electrically heated Cu foil on polyimide substrate [1]. Already here local heat sources in an electronic device have been imaged. Later on LIT became more popular for non-destructive testing (NDT) of constructional materials like airplane components, since photo-thermal investigations allow to "look below the surface" of such components [2]. Other applications of LIT in NDT were thermoelastic [3] and ultrasonic lock-in thermography (ULT [2]). Only after 2000, LIT was applied systematically again for failure analysis in electronic components like solar cells and integrated circuits (ICs) [4]. While in NDT certain inhomogeneities of the investigated material are in focus (e.g. delaminations, missing weld connections, or hidden cavities), the main goal of electronic device testing (EDT) is the detection of local heat sources in a device operating or under specific electronic test conditions. The success of LIT for EDT relies on the fact that all these devices generate heat in operation, hence they contain local heat sources. If there are certain faults in these devices, these faults usually lead to a change in the distribution or in the generation of new local heat sources. Therefore, already before the advent of LIT, conventional (steady-state) thermography has been a standard tool for characterizing electronic components. However, since the noise limit of conventional thermography is in the range of $20 \ldots 100 \mathrm{mK}$, this technique allowed only the detection of relatively strong local heat sources. Moreover, due to the lateral heat conduction in solid devices, local heat sources generally appear blurred in steady-state thermography. These two limitations are strongly improved if LIT is applied instead of conventional thermography in EDT. Due to the averaging nature of LIT, the detection sensitivity lowers to $100 \mu \mathrm{K}$ temperature modulation amplitude and below, and, due to its dynamic nature, lateral heat conduction is strongly suppressed. Moreover, LIT provides easy means of local emissivity correction, in particular for microscopic IC investigations.

In this contribution the application of LIT to EDT will be reviewed. Currently the two main application fields are the shunt imaging and local efficiency analysis of solar cells and failure analysis on other electronic devices, in particular microscopic LIT investigations in ICs and more macroscopic investigation of faults, like internal shorts in encapsulated ICs, printed circuit boards (PCBs), and other electronic components.

\section{Basics of lock-in thermography for electronic device testing}

Though commercial ILIT systems, like the PV-LIT system of InfraTec, can be used both for NDT and EDT, there are some particularities for LIT application to EDT. For example, LIT for EDT uses as a rule synchronous correlation, hence a well-defined number of camera frames is evaluated in each lock-in cycle [4]. For NDT the IR camera cannot always be synchronized to the lock-in process, but for EDT the pulse trigger can easily be derived from the camera frame trigger. While in NDT often sinusoidal modulation of the heat sources is applied, in EDT usually square pulse modulation is used, since here we are interested to operate an electronic component at a well-defined supply voltage. The evaluation of only the basic harmonic of the temperature- ( $T$-) modulation is ensured by applying sin/-cos correlation [4]. There are several possibilities to perform a LIT experiment on an electronic device. In simplest case a well-defined supply voltage (bias) of this device is periodically switched on and off. Some devices lose their operation status if they are completely switched off. Then their operation voltage is modulated between two different biases. For integrated circuits like microprocessors it is also possible to keep the operation voltage constant and to modulate the internal operation periodically by using a programmable IC testing station. For solar cells also pulsed or steady-state light can be irradiated, instead of or in addition to bias modulation. These experiments are called illuminated LIT (ILIT) [4]. Another difference to LIT for NDT is that for EDT not only the amplitude and phase images are evaluated, which is standard in NDT, but also the primary in-phase $\left(0^{\circ}\right)$ and out-of-phase $\left(-90^{\circ}\right)$ images are of interest. Fig. 1 shows all these images resulting from one LIT measurement of a 
multicrystalline silicon solar cell. If the amplitude image is scaled to the maximum signal, as in image (a), only the strongest local heat source in the upper left corner becomes clearly visible. If the same data are displayed with increased contrast (b), many other local heat sources (in particular in the edge region) and also the homogeneous heating in the whole area become visible. The phase image (c) represents the time delay between the power modulation and the resulting temperature modulation. For isolated lying point-like heat sources this phase signal is independent of the power of the source. Therefore in the phase image (c) local heat sources of different magnitude appear with nearly the same brightness. This may be called a "dynamic compression" feature of the phase signal in EDT. Another property of the phase signal, which is also known from NDT, is its independence of the local IR emissivity. The in-phase $\left(0^{\circ}\right)$ signal in Fig. 1 (d,e) shows the best spatial resolution of all LIT signals and is therefore most appropriate for imaging point-like heat sources. This is best visible in image (e) showing increased contrast. Outside of these local heat sources, however, the $0^{\circ}$ signal shows an overshoot into the negative. Homogeneous heat sources remain completely invisible in the $0^{\circ}$ signal of a solar cell, since for them the temperature modulation occurs at a phase of $-90^{\circ}$ [4]. In EDT the $-90^{\circ}$ signal (f) is always displayed instead of the $+90^{\circ}$ signal, since the latter is always negative because the $T$ modulation is more or less delayed to the power modulation (delay means negative phase). This $-90^{\circ}$ signal shows the worst spatial resolution of all LIT images. Its spatial resolution is in the order of the thermal diffusion length, which is about $2 \mathrm{~mm}$ for a silicon device pulsed at $10 \mathrm{~Hz}$ and reduces with $1 / \sqrt{ } f_{\text {lock-in }}$ [4]. However, the $-90^{\circ}$ signal displays both local and homogeneous heat sources and is therefore best appropriate for displaying the local dissipated power density, see Sect. 3.2. If integrated circuits (ICs) are investigated (see Sect. 4), except of the LIT images always a topography image must be taken, which is just a single IR image of the unpowered device. Then there must be the option to superimpose the topography and the LIT image, since only then a local fault can be attributed to a certain position in the the layout of the IC. The PV-LIT system of InfraTec, which is particularly appropriate for EDT, implies all these options.
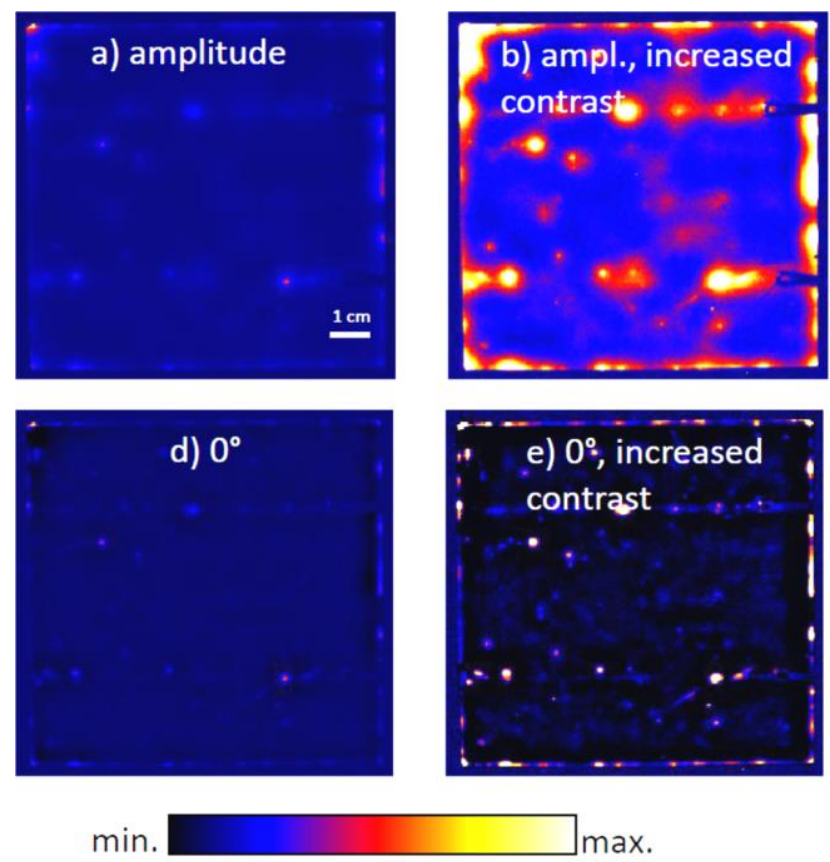
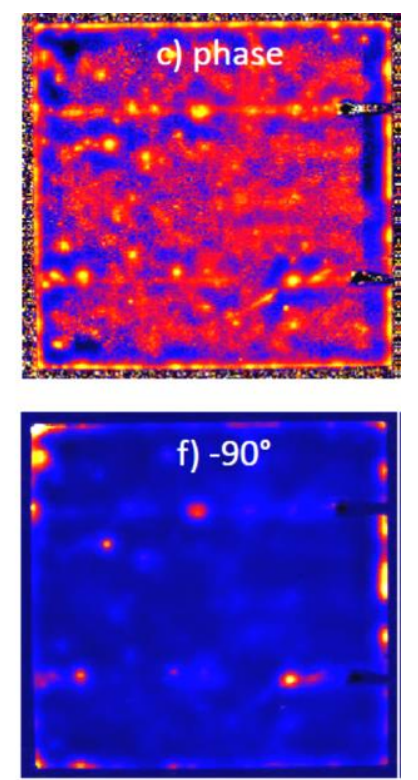

Fig. 1. Different ways to display LIT results of a solar cell, see text.

\section{Lock-in thermography for solar cell analysis}

\subsection{LIT for shunt imaging}

LIT was first applied to solar cells for imaging so-called shunts. Fig. 2 shows schematically two dark (top) and two illuminated current-voltage ( $I$ - $V$ ) characteristics (bottom, red) of solar cells, with and without shunts, all under forward bias. In the dark, positive voltage (forward bias) leads to positive currents, hence the cell dissipates power. The dark characteristic is essentially exponential, hence for low biases the dark current is negligibly small and then it strongly increases with increasing forward bias. It is often assumed that the dark current flows homogeneously in solar cells, but this is as a rule not true. Nearly all solar cells contain local positions showing a strongly increased dark current. These positions are traditionally called "shunts". The bright spots visible in Fig. 1 are typical shunts. These shunts may show a linear (ohmic) or a diode-like characteristic, but they generally tend to increase the dark forward current as shown in Fig. 2 at the top. If a solar cell is illuminated, in addition to the voltage-dependent dark current the photocurrent flows. This photocurrent is by nature a reverse (negative) current and it is essentially voltage-independent. Therefore the illuminated characteristic of a solar cell can be described in good approximation as the dark characteristic shifted to negative current values by a constant current, which is the so-called short circuit current $l_{\text {sc. }}$. This transformation is called the superposition 
principle, since the positive dark current and the negative photocurrent linearly superimpose. Under illumination, the current may flow out of the cell, hence it is negative for positive biases and the cell produces electric power. The working point, where the maximum possible power $P$ can be extracted, is the so-called maximum power point (mpp). At this maximum power point the product of generated current $I_{\mathrm{mpp}}$ and voltage $V_{\mathrm{mpp}}$ is maximum, which is symbolized in Fig. 2 by the rectangular areas. If there are shunts in the cell, both the dark and the illuminated characteristic change, as visible in Fig. 2. Then $I_{\mathrm{mpp}}$ and $V_{\mathrm{mpp}}$ reduce, leading to a reduced generated power $P$ and thus to a reduced energy conversion efficiency of the cell. Therefore all shunts are detrimental for obtaining a good cell efficiency and must be detected and, if possible, avoided. LIT performed in the dark (DLIT) is the technique of choice for shunt imaging in solar cells. Once the positions of shunts have been found, their physical nature can be investigated by applying both LIT and other physical and microscopic investigations.

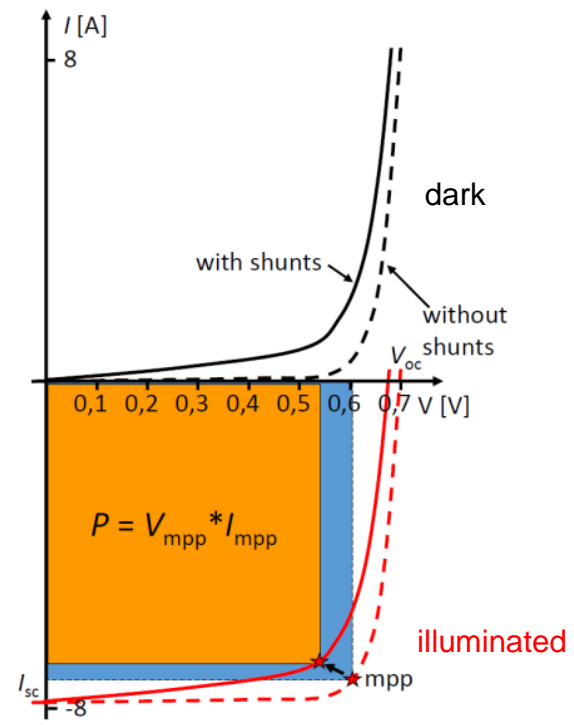

Fig. 2. Dark I-V characteristics (top) and illuminated characteristics (bottom) of a solar cell with and without shunts, see text.
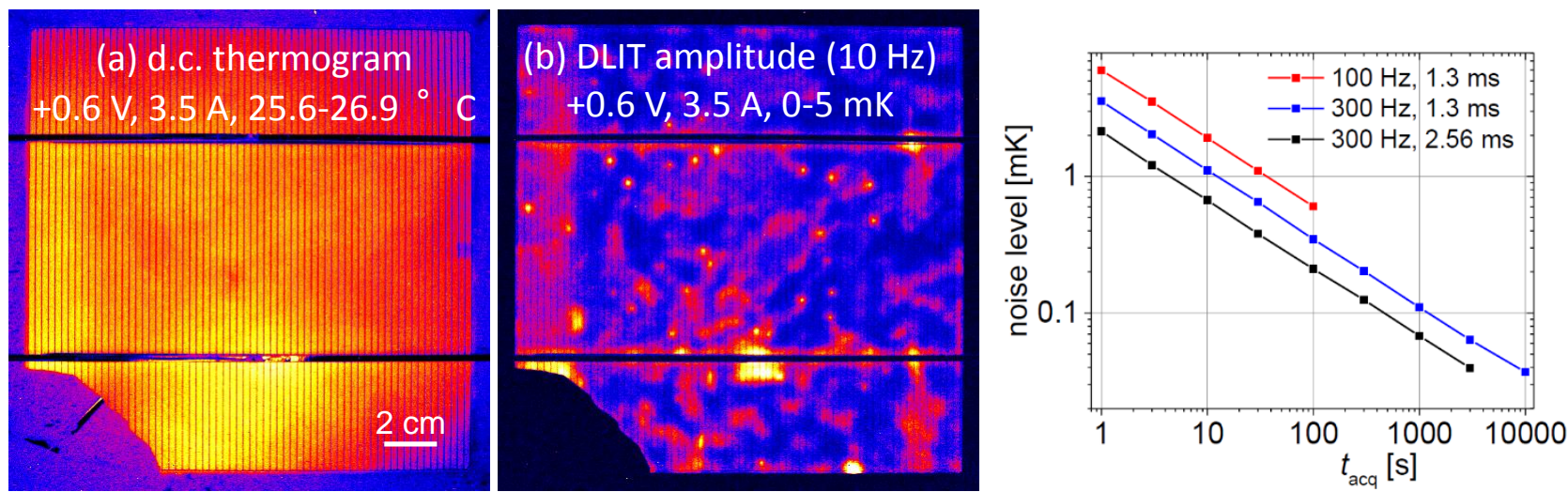

Fig. 3. (a) Steady-state (d.c.) thermogram and (b) dark lock-in thermography image of a solar cell under identic conditions, (c) measured amplitude noise levels of the InfraTec PV-LIT system.

In principle, local shunts could also be made visible in solar cells by applying standard (steady-state) thermography. Indeed, standard thermography is a proven tool for characterizing large solar modules [5]. However, in single solar cells only very strong shunts could be detected, since the sensitivity even of the best modern thermocameras is only in the order of $20 \mathrm{mK}$. This is enough for detecting shunts under higher reverse bias of, say, -10 V, but not for detecting shunts under forward bias, which is most interesting since under operation the cell is forward biased by about $0.55 \mathrm{~V}$, see Fig. 2. Moreover, under steady-state condition the surface temperature profile around local shunts appears very blurred, since the heat tends to diffuse away laterally from the heat sources. Note that silicon is a quite good heat conductor. For LIT, on the other hand, the image noise is greatly reduced by averaging over many lock-in periods, and lateral heat diffusion is suppressed by the dynamic nature of the technique. Note that heat flow in a body takes time. Therefore, LIT can detect orders of magnitude more and weaker local shunts than steady-state thermography can do, which is illustrated in Fig. 3. Here in (a) a standard (d.c.) thermogram of a solar cell at a relatively high forward bias and current is shown. We see that indeed the heat generation seems to be inhomogeneous and has a maximum in the middle of the lower contact stripe (busbar). The DLIT image (b) taken under the same biasing conditions shows this site and a large number of local shunts well-separated from each other. We see that, for the lock-in frequency of $10 \mathrm{~Hz}$ used here, 
the shunts show a T-modulation amplitude in the low $\mathrm{mK}$ range, which is typical. For LIT lateral heat conduction leads only to certain halos around the local shunts. The spatial extension of these halos can be reduced by increasing the lock-in frequency, but this goes on cost of the DLIT signal height, hence it would degrade the signal-to-noise ratio. Hence, the choice of the lock-in frequency represents a compromise between high detection sensitivity (low frequency) and high spatial resolution (high frequency). Fig. 3 (c) shows the measured amplitude noise signal (objective cap closed) of the PV-LIT system of InfraTec (640 x 512 pixel InSb detector, $512 \times 512$ pixels used) as a function of acquisition time for different camera frame rates and frame integration times. The higher the frame rate and the frame integration time, the lower is the noise level [4]. At $t_{\text {acq }}=1000 \mathrm{~s}$, which is below 17 minutes, a noise level below $100 \mu \mathrm{K}$ may be obtained.
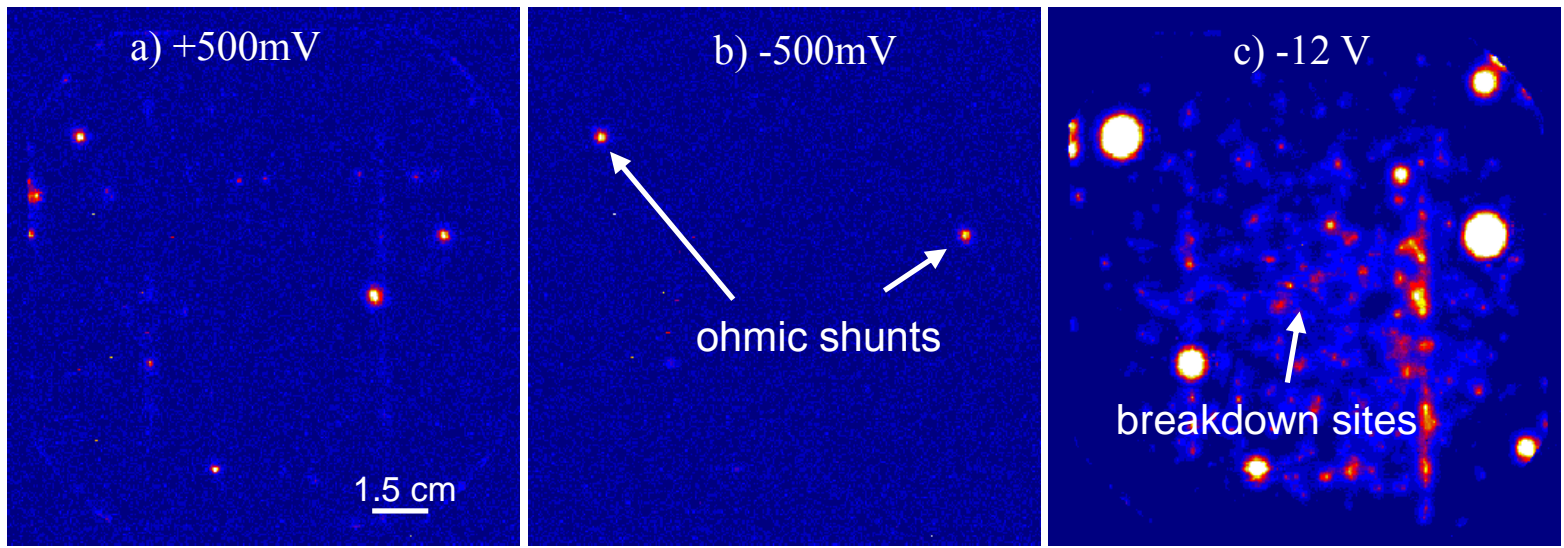

Fig. 4. (a) Forward bias and (b) low reverse bias LIT amplitude image showing shunts with linear and non-linear characteristic, (c) high reverse bias amplitude image showing in addition breakdown sites.

The amount and the polarity of the pulsed bias applied to a solar cell in a DLIT experiment allow to draw conclusions about the physical nature of the detected shunts. This is demonstrated in Fig. 4 showing DLIT images of a solar cell under $500 \mathrm{mV}$ forward bias (a), $-500 \mathrm{mV}$ reverse bias (b), and $-12 \mathrm{~V}$ reverse bias (c). Only the shunts visible under forward and low reverse bias with the same brightness are ohmic shunts showing a linear $I-V$ characteristic. All shunts appearing only under forward bias show a non-linear (diode-like) $I-V$ characteristic. At higher reverse bias (c) local breakdown sites become active, which is a different conduction mechanism than that under low forward and reverse bias.

\subsection{Quantitative evaluation of LIT images of solar cells}

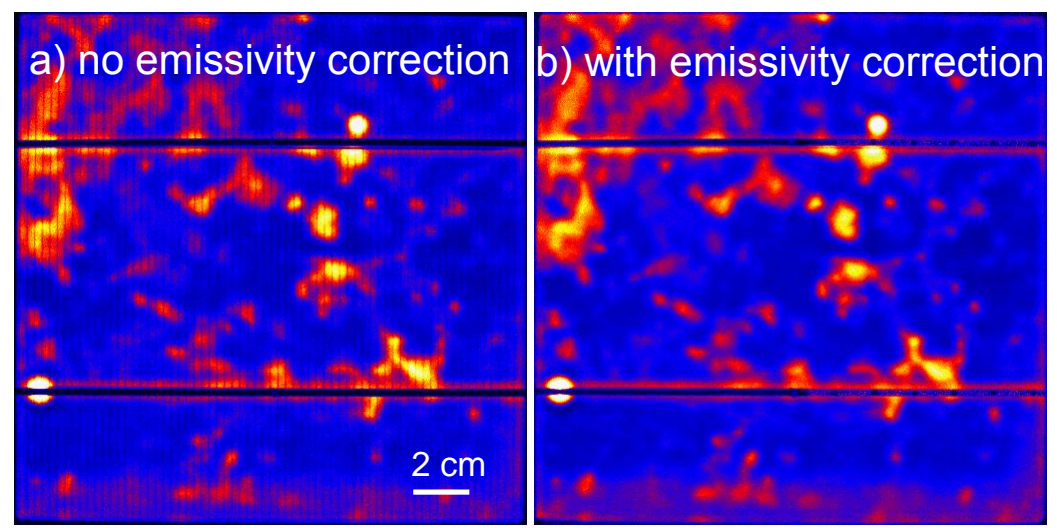

Fig. 5. LIT amplitude images of a bare silicon solar cell (a) without and (b) with local emissivity correction.

The silicon solar cells to be investigated by LIT consist of a $180 \mu \mathrm{m}$ thick sheet of silicon. At a typical lock-in frequency of $10 \mathrm{~Hz}$ the thermal diffusion length is about $2 \mathrm{~mm}$, hence this device can be considered as thermally thin [4]. This silicon material is thermally homogeneous. The metallic gridlines, which are used for feeding away the current, are only $10 \ldots 20 \mu \mathrm{m}$ thick. Hence they do not influence the thermal properties seriously and can be penetrated easily by the thermal waves. However, their IR emissivity is significantly lower than that of the bare cell surface. Therefore, if bare solar cells are imaged by LIT, the gridlines always appear dark as could already be seen in Fig. 3 (b). In was mentioned in Sect. 2 that the $-90^{\circ}$ signal is proportional to the locally dissipated power density. This only holds correct if the emissivity is homogeneous. The usual possibility to homogenize the emissivity is to cover it by black paint. For solar cells, which have to convert light into electric energy, this is usually not wanted. One other possibility is here to suck-on by vacuum a $20 \mu \mathrm{m}$ thin black plastic foil, as it is used for packaging purposes [4]. However, for frequencies of $10 \mathrm{~Hz}$ and above this foil leads already to a significant damping of the thermal waves. Here software-controlled local emissivity correction can be applied. The PV-LIT system of InfraTec provides the possibility to capture thermograms of the un-powered device at two 
temperatures and to calculate an image of the local emissivity, which is used to correct the LIT images. Reflected light needs not to be corrected in LIT since it is not modulated. In Fig. 5 two LIT amplitude images are compared, one taken without and one with local emissivity correction. The emissivity-corrected image is best appropriate for quantitative evaluation, but also by using the un-corrected images the resulting quantitative error is only weak since the gridlines occupy only a small fraction of the area.

It was mentioned in Sect. 2 that the $0^{\circ}$ image shows a sharp maximum in the position of local shunts and becomes negative in greater distance to the shunts, its average over the area is zero. The LIT amplitude image, which is always positive, is the vectorial sum of the $0^{\circ}$ and the $-90^{\circ}$ images. For several nearby lying shunts the horizontally running thermal waves superimpose. Then only the vectorial components $\left(0^{\circ}\right.$ and $-90^{\circ}$ images) superimpose linearly, but not the amplitude signals, since the amplitude is a non-linear combination of the vectorial components. Therefore the amplitude signal is less appropriate for quantitative evaluation, since it would overestimate the signal close to local shunts. As already mentioned in Sect. 2, the local $-90^{\circ}$ signal $S^{90^{\circ}}(x, y)$ is best appropriate for quantitative evaluation, since, in the limit of its low spatial resolution, it is strictly proportional to the locally dissipated heat. Note that each LIT system shows a systematic phase error due to the processing times needed for the digital signal processing. For performing quantitative LIT evaluation this phase error must be able to be corrected. This can be done by zeroing the averaged $0^{\circ}$ signal; the PV-LIT system of InfraTec contains an automatic option for this. The proportionality factor between the $-90^{\circ}$ signal and the local power density is found by evaluating the average of the $-90^{\circ}$ signal over the whole solar cell $\left\langle S^{90^{\circ}}\right\rangle$, which must be proportional to the whole dissipated power (product of measured voltage $V$ and current $I$ during the excitation pulses). This leads to the following simple formula for converting $-90^{\circ} \mathrm{LIT}$ images to images of the dissipated power density $p(A=$ cell area):

$$
p(x, y)=\frac{S^{-90^{\circ}}(x, y) V I}{A<S^{-90^{\circ}}>}
$$

Note that this formula is "self-calibrating", hence it needs neither the thermal parameters of the object nor any sensitivity parameter of the IR camera. Based on this formula the software program "Local I-V 2" for evaluating LIT images has been developed for performing the so-called "Local I-V" method, which is available [6]. This software evaluates up to four $-90^{\circ}$ DLIT images, three measured at different forward biases and one measured under weak reverse bias, where no breakdown processes are expected. As its name says, the "Local I-V" method is used for non-destructively (thermally) measuring local I-V characteristics of solar cells. This method and its application to typical solar cells is described in detail in $[7,8]$, therefore only its basic principles and some selected results will be reported here. The software first converts all entered LIT images into local power density images by using (1). Then it converts these images into images of the local current density and the local diode voltage by considering the effective local series resistance $R_{\mathrm{s}}(\mathrm{x}, \mathrm{y})$. This series resistance may be assumed to be homogeneous, an $R_{\mathrm{s}}$ image may be imported e.g. from luminescence measurements [9], or a luminescence-measured local diode voltage image is imported and evaluated together with LIT results as described in [10]. The local dark current densities are then fitted for each image pixel separately to the so-called two-diode model, which is the standard model for describing dark and illuminated characteristics of solar cells $[4,7]$. Once the parameters of the two-diode model for each pixel are known, the software calculates local dark and illuminated characteristics for each pixel by using the superposition principle described in Sect. 2. Fig. 6 shows a selection of $I-V$ characteristics measured thermally by "Local I-V" in four different positions of a solar cell. There are three physically different dark current contributions in a solar cell, which are the diffusion current $J_{\text {diff, }}$ which is the nominal diode current, the depletion region recombination current Jrec flowing only in certain shunt positions, and the ohmic shunt current, which can be due to local material defects or technological faults. The diffusion current shows the fastest increasing exponential characteristic, the recombination current a weaker increasing exponential characteristic, and ohmic shunts show a linear characteristic. By knowing these different voltage dependencies, these three components are separated from each other in "Local I-V". The images in Fig. 6 show the dominating components of the dark characteristics, the sum dark characteristics, and the resulting illuminated characteristics in 4 positions of a cell. The black dots are the points where the current densities were measured by DLIT under forward bias. We see that these characteristics differ significantly from each other, pointing to the different physical origins of their dominating components. Based on these local characteristics, "Local I-V" calculates images of important solar cell parameters, like the open circuit voltage $V_{\text {oc, }}$ the fill factor (a measure of the rectangular shape of the illuminated characteristic), or the conversion efficiency [8]. Such investigations are very helpful in the process of looking for physical origins of poor efficiency parameters and are an example par excellence for quantitative IR thermography (QIRT).

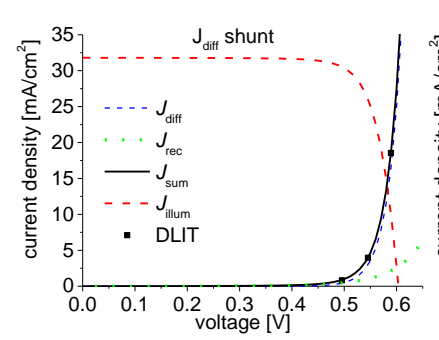

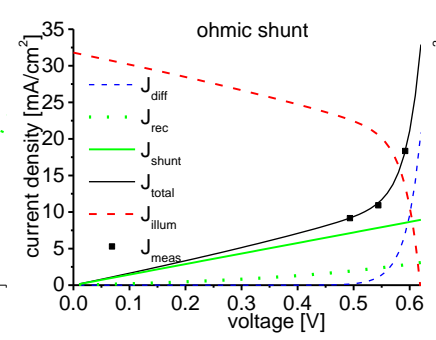

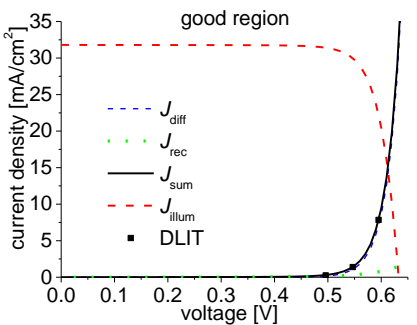

Fig. 6. Thermally measured dark and illuminated local I-V characteristics of different positions in a solar cell (Udiff shunt, Jrec shunt, ohmic shunt, and a good region). 


\section{Lock-in thermography for bare and encapsulated ICs}

Since its introduction to integrated circuit (IC) failure analysis (FA) in 2000 [11], LIT has become a standard method also in this field. The success of LIT for IC FA relies on the fact that many faults in ICs, like internal shorts, latchups, faulty transistors or diodes, or other leakage currents, generate local heat. For these investigations generally midwave IR cameras are used, for which, even with the best available microscope objectives, the diffraction-limited spatial resolution is in the order of $5 \mu \mathrm{m}$. This resolution can be reduced down to about $1.5 \mu \mathrm{m}$ by applying a solid immersion lens [12]. However, the structural dimensions of present ICs are with $<0.1 \mu \mathrm{m}$ still well below the spatial resolution of LIT. Nevertheless LIT is very successful for at least a first coarse localization of faults due to its extreme sensitivity and easy applicability. Once a fault is localized by LIT to an accuracy of a few $\mu \mathrm{m}$, its detailed position may be revealed later on e.g. by light emission microscopy, electron beam methods, laser-based methods, or electric nano-probing methods [13]. Fig. 7 shows a topography image (a) and a LIT amplitude image (b) of an intact IC with the supply voltage pulsed. The bright spots in (b) are due to the normal operation of this IC. Fig. 7 (c) shows the LIT amplitude image of a defect IC of the same type. Here two additional bright spots are visible, which indicate the locations of the faults being responsible for the defect in this IC.

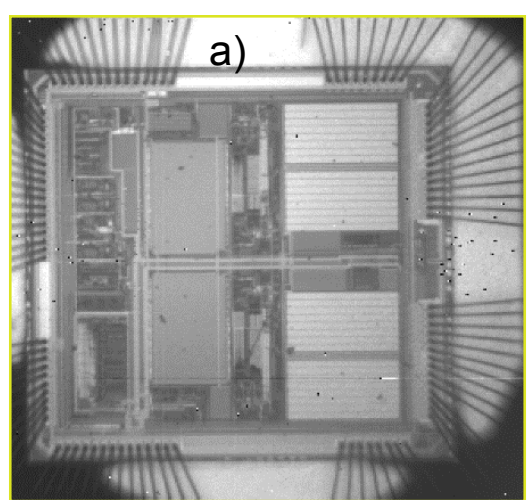

Fig. 7. (a) Topography image and
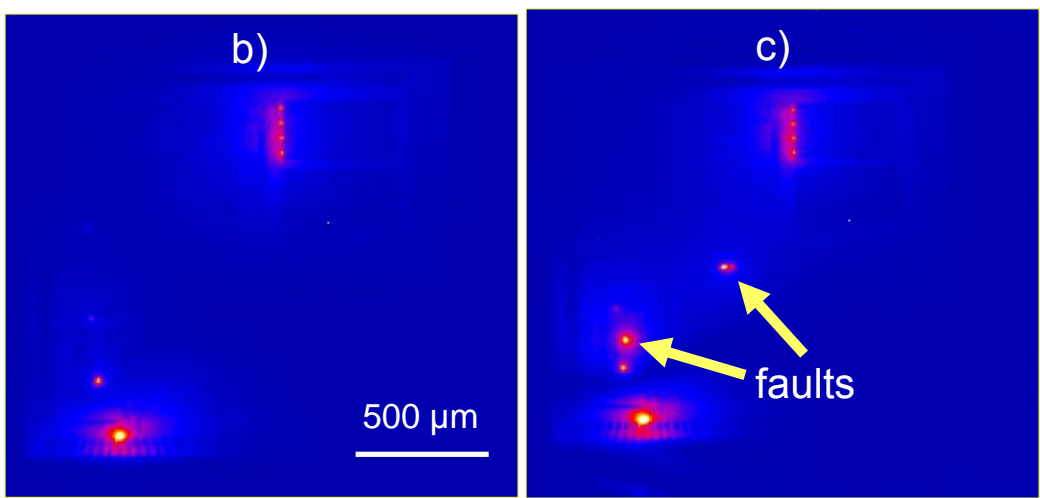

(b) LIT amplitude image of an intact
The arrows point to the fault locations.

a) topography

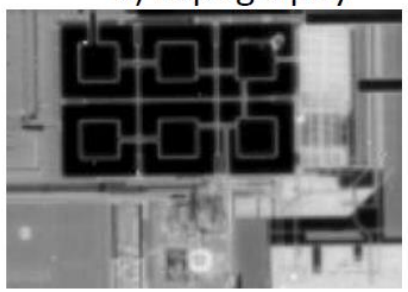

d) phase

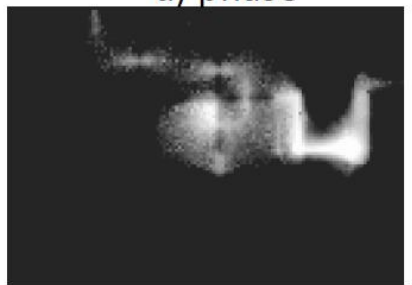

b) $0^{\circ}$

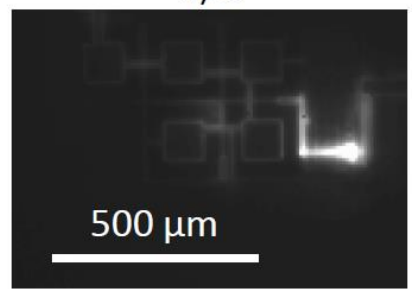

e) $0^{\circ} /-90^{\circ}$

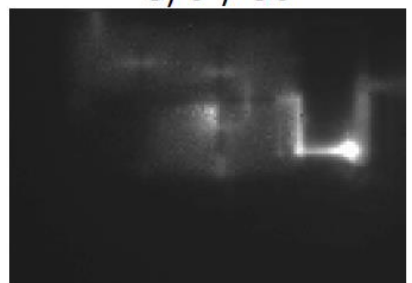

c) $-90^{\circ}$
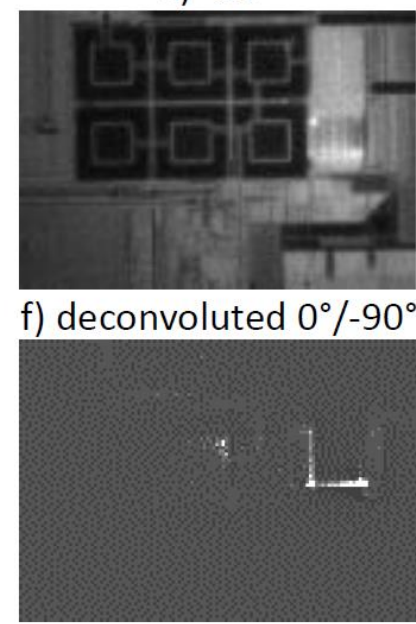

Fig. 8. (a) Topography image, (b) $0^{\circ}$ image, (c) $-90^{\circ}$ image, (d) phase image, (e) $0 \%-90^{\circ}$ image, and (f) spatially deconvoluted $0 \%-90^{\circ}$ image of a region in a bare $I C$.

A particular problem for LIT on bare ICs is the inhomogeneous IR emissivity, which is much lower in metallized than in non-metallized regions. If a magnifying microscope objective is used, the image field is smaller than the detector. Then the whole image field is within the black spot of the "Narcissus effect", hence the metallization appears in topography dark, and not metallized regions appear bright. If the image "wiggles" e.g. due to vibrations caused by the Stirling cooler of the IR camera, on each bright-dark-edge a strong intensity modulation appears, which is interpreted by the lock-in correlation procedure wrongly as a LIT signal. These artifacts can be minimized by choosing a lock-in frequency, which is sufficiently apart from the vibration frequency and its harmonics. An off-line correction of these artifacts is not possible. For avoiding image wiggling, a mechanically strong connection must be established between camera (the objective) and the imaged object. InfraTec provides a very stable camera support with x-y table for performing LIT for IC analysis. 
Even if the image does not wiggle, local emissivity correction as described in Sect. 3.2 is hardly applicable in microscopic regions, since it is hard to avoid microscopic lateral image movement if the device temperature is changed. If the images at the two temperatures are not exactly in the same position, the obtained emissivity image shows strong artifacts on edges of the metallization. Here the display of the $0 \%-90^{\circ}$ signal is very helpful, as it is demonstrated in Fig. 8, see [14]. This figure shows various representations of LIT results of a region in an IC. In the topography image (a) we see the strong emissivity contrast, all regions appearing dark are metallized regions. The $0^{\circ}$ signal (b) shows the best spatial resolution, but it is also modulated by the emissivity contrast. The phase signal (d) does not show any emissivity contrast, but it shows a low spatial resolution and, due to the dynamic compression feature mentioned in Sect. 2, it is not proportional to the dissipated power density. The $-90^{\circ}$ signal (c) shows only a weak maximum in the position of the dominant $0^{\circ}$ signal. This is due to the inherently low spatial resolution of this signal of about $2 \mathrm{~mm}$ here, which was mentioned already in Sect. 2. Hence the $-90^{\circ} T$-modulation is nearly homogeneous in this small region, but the $-90^{\circ} \mathrm{IR}$ image is modulated by the local emissivity. Indeed, the $-90^{\circ}$ signal (c) looks similar to the topography signal (a), but in contrast to this it shows a welldefined zero signal level. Therefore, in micro regions where the thermal diffusion length exceeds the image size, the $-90^{\circ}$ signal can be used as a measure of the local emissivity. Then the $0 \%-90^{\circ}$ signal (e) is an "emissivity-corrected $0^{\circ}$ signal". This signal can even be deconvoluted for improving its spatial resolution (f), which, however, increases the noise level [14].

A more recent development is 3D Analysis of encapsulated stacked ICs [15]. In particular for memory devices it is usual to glue several thin chips (called "dies") on top of each other for increasing the packing density. Then, if the whole device should show a heat-producing fault, it is interesting to know in which of the stacked dies the fault is located. This can be measured by measuring the frequency-dependence of the phase of the temperature modulation at the surface of the mould compound used for encapsulating the IC. If the thermal wave travels vertically from the fault position to the surface of the device, at the junction from each die to die a phase shift occurs due to the heat capacity of the die and the heat resistance of the glue layer between the dies. In the top mould compound an additional phase shift occurs. This is illustrated in Fig. 9 (a). Hence, depending on in which die the fault is located, a different frequency-dependent phase of the temperature modulation at the surface is expected. This can be numerically modelled if the thermal properties and the dimensions of all layers are known. Fig. 9 (b) shows the result of such a measurement together with theoretical calculations of the phase shift for the fault lying in different dies. In this case the fault was lying in die no. 1 close to the surface, leading to the lowest amount of the phase shift.

a)

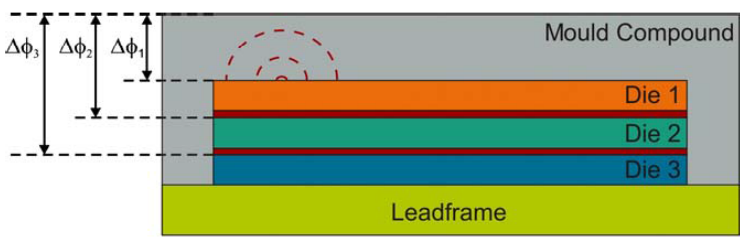

b)

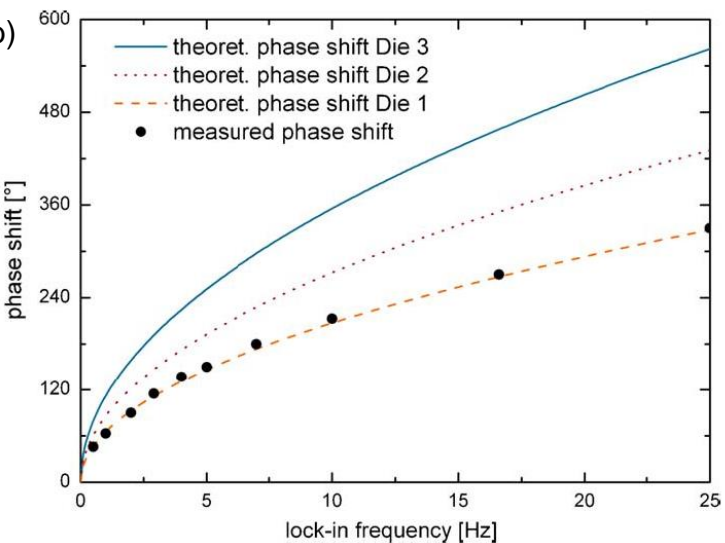

Fig. 9. (a) Cross section through a stacked IC, (b) simulated (theoretical) phase shift for a defect lying in different dies of the IC and measured phase shift values for different lock-in frequencies.

\section{Conclusions}

Lock-in thermography (LIT) has established in the last 15 years as a standard method not only in non-destructive testing (NDT) but also in electronic device testing (EDT). For being useful in EDT, commercial LIT systems must fulfil certain special requirements, like special display options, the possibility to correct systematic phase errors, 4-wire connection to the sample with exact display of the applied voltage, local emissivity correction, and T-drift correction. Until now the main application fields of LIT in EDT are shunt imaging and local efficiency analysis in solar cells and failure analysis in other electronic components like integrated circuits. However, LIT can be used to locate heat sources in any electronic device. If the devices are thermally homogeneous, the results can be evaluated in terms of measured power densities or depth of a heat-producing fault below the surface. For investigating solar cells, the "Local I-V" method provides a powerful tool for performing a detailed quantitative local efficiency analysis. By this analysis local shunts, which are origins of degraded efficiency parameters of a cell, can be found and analysed and their influence on the solar cell efficiency can be determined. For investigating ICs, heat-producing faults can be localized up to an accuracy of $5 \mu \mathrm{m}$ and up to 1.5 $\mu \mathrm{m}$ by using a solid immersion lens [4,12]. With the continuing trend of falling prices of IR cameras, it can be expected that the use of LIT in EDT will further increase.

The authors are grateful to F. Altmann and Ch. Schmidt (Fraunhofer IWM Halle), Jan Bauer and F. Frühauf (MPI Halle), and many other colleagues for experimental cooperation. Part of this work was supported by the German Federal Ministry for Economic Affairs and Energy in the "SolarLIFE" (0325763 D) and foregoing projects. 


\section{REFERENCES}

[1] Kuo P.K., Ahmed T., Jin H., Thomas R.L., "Phase-locked image acquisition in thermography", Proceedings of SPIE 1004, Automated Inspection and High Speed Vision Architectures II, p. 41, 1988.

[2] Busse G., "From photothermal radiometry to lock-in thermography methods". J. Phys. Conf. Ser. - Vol. 214, 012003 (1-7), 2010.

[3] Harwood N., Cummings W.M. Thermoelastic Stress Analysis. Adam Hilger, Bristol, 1991.

[4] Breitenstein O., Warta W., Langenkamp M., "Lock-in Thermography - Basics and Use for Evaluating Electronic Devices and Materials", Second Edition. Springer, Heidelberg, 2010.

[5] Buerhop Cl., Schlegel D., Niess M., Vodermayer C., Weißmann R., Brabec C.J., "Reliability of IR imaging of PV plants under operating conditions", Solar Energy Mat. \& Solar Cells Vol. 107, pp. 154-164, 2012.

[6] See <www.maxplanckinnovation.de> (April 2018)

[7] Breitenstein O., "Nondestructive local analysis of current-voltage characteristics of solar cells by lock-in thermograpphy", Solar Energy Mat. \& Solar Cells. - Vol. 95, pp. 2933-2936, 2011.

[8] Breitenstein O., "Local efficiency analysis of solar cells based on lock-in thermography", Solar Energy Mat. \& Solar Cells. - Vol 107, pp. 381-389, 2012.

[9] Trupke T., Pink E., Bardos R.A., Abbott M.D., "Spatially resolved series resistance of silicon solar cells obtained from luminescence imaging", Appl. Phys. Lett. Vol. 90, 093506 (1.3), 2007.

[10] Ramspeck K., Bothe K., Hinken D., Fischer B., Schmidt J., Brendel R., "Recombination current and series resistance imaging of solar cells by combined luminescence and lock-in thermography", Appl. Phys. Lett. Vol. 90, 153502 (1-3), 2007.

[11] Breitenstein O., Langenkamp M., Altmann F., Katzer D., Lindner A., Eggers H., "Microscopic lock-in thermography investigation of leakage sites in integrated circuits", Rev. Sci Instr. Vol. 71, no. 11, pp. 41554160.

[12] Breitenstein O., Altmann F., Riediger T., Karg D., Gottschalk V., "Use of a solid immersion lens for thermal IR imaging" Proceedings of 32th Int. Symposium for Testing and Failure Analysis (ISTFA), Austin TX, pp. 382-388, 2006.

[13] Ross R.J. (ed.) Microelectronics Failure Analysis: Desk Reference, Sixth Edition, Ohio, ASM Internationational, 2011.

[14] Breitenstein O., "Lock-in IR thermography for functional testing of solar cells and electronic devices", QIRT Journal Vol. 1, no. 2, pp. 151-172.

[15] Schmidt, Ch., Altmann F., Breitenstein O., "Application of lock-in thermography for failure analysis in integrated circuits using quantitative phase shift analysis", Mat. Sci. and Eng. B, Vol. 177, pp. 1261-1267, 2012. 